\title{
Complex Dynamics of Pell Sequence
}

\author{
Rajeshri Rana \\ Asst. Professor \\ Applied Science and Humanities \\ Department \\ G. B. Pant Engg. College, \\ Pauri Garhwal
}

\author{
Yashwant S Chauhan \\ Asst. Professor \\ Computer Science \& Engg. \\ Department \\ G. B. Pant Engg. College, \\ Pauri Garhwal
}

\author{
Ashish Negi \\ Asst. Professor \\ Computer Science \& Engg. \\ Department \\ G. B. Pant Engg. College, \\ Pauri Garhwal
}

\begin{abstract}
The Binet formula for Pell sequence is viewed as a function of complex variable. In this paper the study of attracting and repelling fixed points of Pell sequence is presented with the complex dynamics resulting in the escape time images. A study of orbits of the Binet type formula is presented in the paper. Besides this, a new class of Mandelbrot sets is also studied for the Mann-iterates.
\end{abstract}

\section{Keywords}

Complex dynamics, Fibonacci sequence, Pell sequence, Binet formula and Binet Type formula.

\section{INTRODUCTION}

The Fibonacci sequence $f_{1}, f_{2}, f_{3}, f_{4} \ldots$ is determined by the equations $f_{0}=0, f_{1}=1$ and for $n \geq 2, F_{n}=a F_{n-1}+b F_{n-2}$. The first two terms of the sequence are equal to one while the subsequent terms are the sum of their two immediate predecessors. It follows as $0,1,1,2,3,5,8,13,21,34, \ldots$ The Fibonacci sequence is a great source for mathematical conjectures and has many remarkable properties. The Fibonacci sequence possesses various mathematical properties. The Binet formula provides a mechanism for the Fibonacci numbers to be viewed as a function of a complex variable. Various properties of Pell Number sequence has been studied by different researchers, see for instance [5], [7] and [8]-[10]. In this paper we have studied the complex dynamics of Pell Number Sequence, its fixed points and their attracting or repelling behavior. Further, the behavior of iteration of the function in the complex plane is also studied in the paper. The purpose of the paper is to investigate complex dynamics of Pell sequence. We have also applied Mann-iteration for studying the dynamics of Pell function. Perhaps the Mandelbrot set is the most popular object in the fractal theory and has been the subject of intense research right from its advent. Our study reveals some beautiful escape time fractals images for the modified Fibonacci sequence i.e. Pell sequence.

\section{Preliminaries}

Definition 2.1: Generalized Fibonacci sequence: Let $a$ and $b$ be any real numbers. Define sequence $F_{n}$ as, $F_{n+2}=a F_{n+1}+b F_{n}$. Such a sequence is described as
Generalized Fibonacci sequence. For $\mathrm{a}=\mathrm{b}=1$, the sequence gives the Fibonacci sequence.

Definition 2.2: Pell Number Sequence: In real plane coordinates system, for any fixed $a$ and $b$, denoted by $R(a, b)$, we have the set of all real sequences. In $R(a, b)$, we have two distinguished elements 0 and 1 . A general case of $\mathrm{R}(1,1)$ is the Fibonacci sequence. Generally, we denote it by $F a, b$. A very interesting sequence is generated for $\mathrm{R}(2,1)$, the sequence is obtained is known as Pell sequence see [9]. The sequence is given as $P=0,1,2,5,12,29,70, \ldots$, particularly denoted for any $n$, that gives a solution to Pell's equation $x^{2}-d y^{2}=1$, where $d=k^{2}+1$. For, further details about the Pell and PellLucas numbers one may refer to Bicknell [2] and Melham [11].

Definition 2.3: Binet Formula: An alternative way of representing Fibonacci sequences is the famous Binet formula, which was first discovered by Euler and later on modified by Binet in 1843.

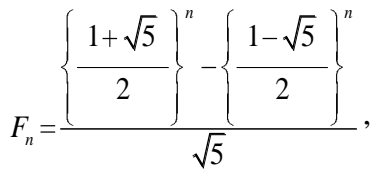

for every $n=1,2,3,4, \ldots$

This gives $F_{n}$ explicitly for every positive integer $n$, without referring to the earlier terms.

Hence, this formula is given in terms of three irrational numbers:

$$
a=\frac{1+\sqrt{5}}{2}, b=\frac{1-\sqrt{5}}{2} \text { and } c=\sqrt{5}
$$

Definition 2.4: Binet Type Formula: If, we take $R(2,1)$, the Pell sequences are generated, for which the roots of the equation $t^{2}-2 t-1$ are $(1 \pm \sqrt{2})$.Hence we have the following formulas:

Formula 1: The Binet type formula for Pell-Fibonacci sequence evolves as follows:

$$
F_{n}=\frac{(1+\sqrt{2})^{n}-(1-\sqrt{2})^{n}}{2 \sqrt{2}}
$$


where $a=(1+\sqrt{2}), b=(1-\sqrt{2})$ and $c=2 \sqrt{2}$.

Formula 2: The Binet type formula for Pell-Lucas sequence evolves as follows:

$$
L_{n}=(1+\sqrt{2})^{n}+(1-\sqrt{2})^{n}
$$

where $a=(1+\sqrt{2})$ and $b=(1-\sqrt{2})$.

Definition 2.5: Mann-Iteration: Let $\mathrm{z}_{0}$ be any arbitrary element of C. Construct a sequence $Z_{n}$ of points of $C$ in the following manner: $\mathrm{z}_{\mathrm{n}}=\mathrm{s} \cdot f\left(\mathrm{z}_{\mathrm{n}-1}\right)+(1-s) \cdot \mathrm{z}_{\mathrm{n}-1}, \mathrm{n}$ $=1,2,3 \ldots$ where the parameters lies in the closed interval $[0,1]$ and $\mathrm{C}$ is the complex plane.

\section{Fixed Points:}

The fixed points of $F(z)$ are those values of $z$, for which $F(z)=z$ while we are applying Formula 1 . For this purpose we will observe several values of $z$ and calculated $F(z)$ :

Table 1: Calculation of $F(z)$ of Pell- Fibonacci for different values of $z$

\begin{tabular}{|c|c|c|c|c|c|c|c|c|c|c|c|}
\hline$z$ & -5 & -4 & -3 & -2 & -1 & 0 & 1 & 2 & 3 & 4 & 5 \\
\hline$F(z)$ & 29 & -12 & 5 & -2 & 1 & 0 & 1 & 2 & 5 & 12 & 29 \\
\hline
\end{tabular}

So, we noticed that $-2,0,1$ and 2 acted as fixed points for Pell-Fibonacci sequence. We also observe that as the values of $F(z)$ changes from negative to positive between -4 and -3 , so there happens to be another fixed point but as definition of $F(z)$ involves exponential with negative base, so we will obtain complex values of $F(z)$ at intermediate values. So, there happens to be many complex fixed points.

On other hand, while we are having comparative study for Pell-Lucas sequence, so, we will apply Formula 2 for finding out values of $z$ and calculated $L(z)$ as follows:

Table 2: Calculation of $L(z)$ of Pell- Lucas for different values of $z$

\begin{tabular}{|c|c|c|c|c|c|c|c|c|c|c|c|}
\hline$z$ & -5 & -4 & -3 & -2 & -1 & 0 & 1 & 2 & 3 & 4 & 5 \\
\hline$F(z)$ & -82 & 34 & -14 & 6 & -2 & 2 & 2 & 6 & 14 & 34 & 82 \\
\hline
\end{tabular}

Here we notice that there is no existence of any fixed point for Pell-Lucas sequence in real axis. But since the value of changes from negative to positive between -1 and $0,-3$ and $-2,-5$ and -4 and so on, such that there happens to be infinitely many fixed point for this function $L(z)$ on the negative axis but the nature of fixed points is purely complex in nature.

\section{Plotting of the points:}

(i) Graph of Pell-Fibonacci function

Fig 1: The graph of fixed points of $F(z)$ and $z$ as shown in Table 1

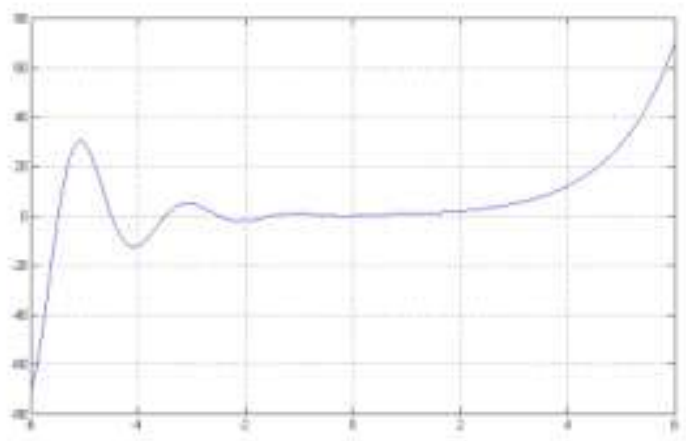

Fig 2: The fixed points of $F(z)$ and $z$ of Table 1, represented by arrows

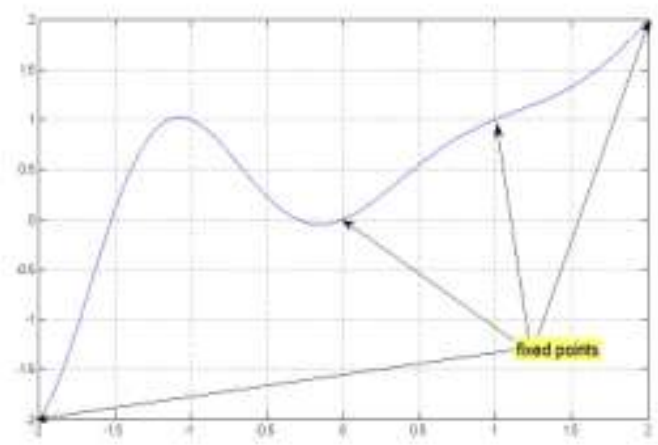

The figure above shows that for $F(z)-z$, the points $-2,0,1$ and 2 are the zeroes or the fixed points for Pell-Fibonacci function and for this it appears that there are no other real fixed points. We can determine the situation off the real axis by looking a dynamics of $F(z)-z$. Fig (7) shows that a plot where $\mathbf{- 0 . 9 1} \leq \boldsymbol{R} \boldsymbol{e}(z), \boldsymbol{I m}(z) \leq \mathbf{- 1 . 0 7}$. The lowest points are black colored while the higher points represent hues running from red to magenta. Besides this, we observe the large black region in the upper right quadrant and some other black regions around the centre, which suggests that there is the existence of infinitely many fixed points in complex plane.

Derivatives of the function $F(z)$ :

The table below shows the derivatives of the function $F(z)$ and its magnitude for the fixed points observed in the Table 1.

Table 3. The derivatives at fixed points for various values of $z$ for Table 1.

\begin{tabular}{|c|c|c|}
\hline$z$ & $F^{\prime}(z)$ & $\left|F^{\prime}(z)\right|$ \\
\hline $\mathbf{( - 2 , 0 )}$ & $-0.049017702+1.986122869 \boldsymbol{i}$ & 1.992162363 \\
\hline $\mathbf{( 0 , 0 )}$ & $2.108531764-1.482027395 \boldsymbol{i}$ & 2.77294956 \\
\hline $\mathbf{( 1 , 0 )}$ & $0.8226790299+0.1183769165 \boldsymbol{i}$ & 0.8311521405 \\
\hline $\mathbf{( 2 , 0 )}$ & $1.986122869-0.04901717002 \boldsymbol{i}$ & 3.96871088 \\
\hline
\end{tabular}


We observe that the magnitude of derivative at $z=(1,0)$ is less than 1 , which shows the existence of attracting fixed point, while the magnitude of derivative at $z=(-2,0),(0,0)$ and $(2,0)$ are greater than 1 , so they represent the repelling points. The set of points that do not diverge to infinity are the filled Julia sets. When the Julia set is nontrivial, there happens a view that such sets with an escape time shows how quickly points outside the Julia set get large.

\section{(ii) Graph of Pell- Lucas function:}

Fig 3: The graph of fixed points of $L(z)$ and $z$ as shown in Table 2

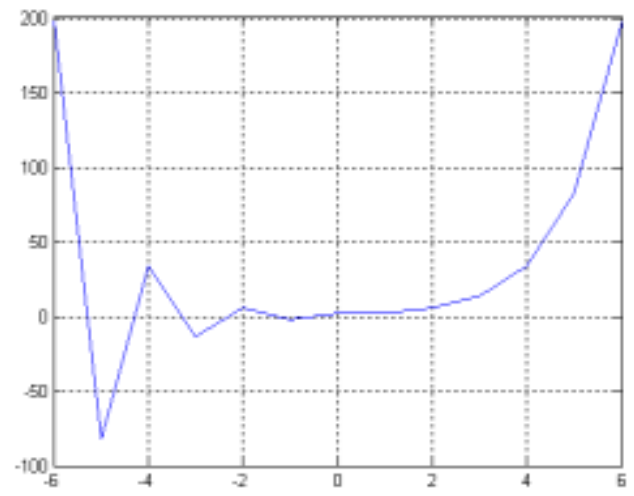

In this graph, we observe that none of the fixed points are lying in the real axis. So, the complex dynamics of Pell-Lucas as shown in Figure (9) represents no darker or black colored region as the fixed points, although the calculations done in the Table 2 proves the existence of the fixed points but they are hence purely complex or imaginary. Thus, the Pell-Lucas dynamics shows less multicolored regions

\section{Iterations of Pell- Fibonacci function $F(z)$ at different points:}

Table 4. Orbit of $F(z)$ for $\left(z_{0}=0.8226790299+0.1183769165 i\right)$

\begin{tabular}{|c|c|c|c|}
\hline $\begin{array}{c}\text { Number of } \\
\text { iteration } \boldsymbol{i}\end{array}$ & $|\mathbf{F}(\mathbf{z})|$ & $\begin{array}{c}\text { Number of } \\
\text { iteration } \boldsymbol{i}\end{array}$ & $|\mathbf{F}(\mathbf{z})|$ \\
\hline 1 & 0.8192 & 21 & 0.9988 \\
\hline 2 & 0.8753 & 22 & 0.9994 \\
\hline 3 & 0.9739 & 23 & 1 \\
\hline 4 & 1.0536 & 24 & 1.0003 \\
\hline 5 & 1.0717 & 25 & 1.0004 \\
\hline 6 & 1.0547 & 26 & 1.0003 \\
\hline 7 & 1.028 & 27 & 1.0001 \\
\hline 8 & 1.0036 & 28 & 1 \\
\hline 9 & 0.9877 & 29 & 0.9999 \\
\hline 10 & 0.9821 & 30 & 0.9999 \\
\hline 11 & 0.9848 & 31 & 0.9999 \\
\hline 12 & 0.992 & 32 & 1 \\
\hline 13 & 0.9993 & 33 & 1 \\
\hline 14 & 1.0039 & 34 & 1 \\
\hline 15 & 1.0053 & 35 & 1 \\
\hline 16 & 1.0042 & 36 & 1 \\
\hline 17 & 1.0021 & 37 & 1 \\
\hline
\end{tabular}

\begin{tabular}{|l|l|l|l|}
\hline 18 & 1.0001 & 38 & 1 \\
\hline 19 & 0.9989 & 39 & 1 \\
\hline 20 & 0.9985 & 40 & 1 \\
\hline
\end{tabular}

Fig. 4: Orbit of $F(z)$ at $\mathrm{z}_{0}=\mathbf{0 . 8 2 2 6 7 9 0 2 9 9}+0.1183769165 i$

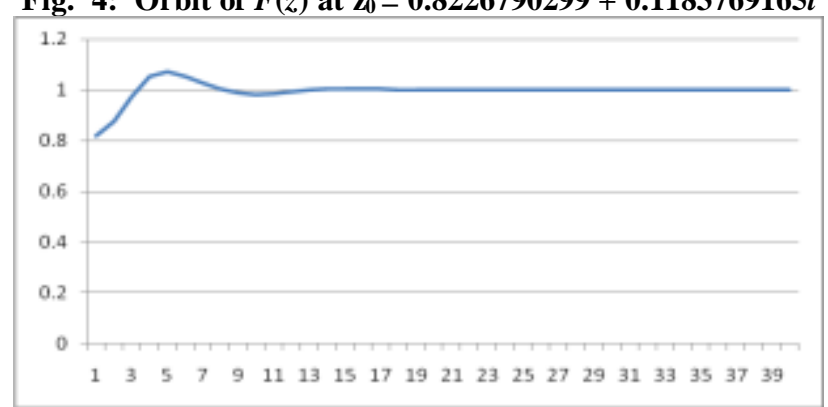

Here we observe that the value converges to a fixed point after 32 iterations

Fig. 5: Orbit of $F(z)$ at $\mathrm{z}_{0}=\mathbf{- 0 . 0 4 9 0 1 7 7 0 2}+\mathbf{1 . 9 8 6 1 2 2 8 6 9 i}$

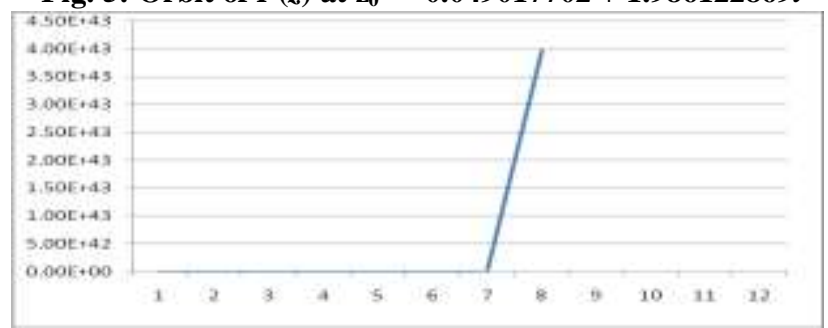

Here we observe that the value escapes to infinity after a few iterations

Table 5: Orbit of $F(z)$ for $\mathrm{z}_{0}=-\mathbf{0 . 0 4 9 0 1 7 7 0 2}+1.986122869 i$.

\begin{tabular}{|c|c|}
\hline $\begin{array}{c}\text { Number of } \\
\text { Iteration } \mathbf{i}\end{array}$ & $|\mathbf{F}(\mathbf{z})|$ \\
\hline 1 & $3.39 \mathrm{E}-01$ \\
\hline 2 & $2.59 \mathrm{E}-01$ \\
\hline 3 & $2.64 \mathrm{E}-01$ \\
\hline 4 & $3.42 \mathrm{E}-01$ \\
\hline 5 & $6.03 \mathrm{E}-01$ \\
\hline 6 & $2.07 \mathrm{E}+00$ \\
\hline 7 & $2.18 \mathrm{E}+02$ \\
\hline 8 & $3.96 \mathrm{E}+43$ \\
\hline
\end{tabular}

Table 6: Orbit of $F(z)$ for $\mathrm{z}_{0}=-0.1+-0.4 i$

\begin{tabular}{|c|c|c|c|}
\hline $\begin{array}{c}\text { Number of } \\
\text { iteration } \boldsymbol{i}\end{array}$ & $|\mathbf{F}(\mathbf{z})|$ & $\begin{array}{c}\text { Number of } \\
\text { iteration } \boldsymbol{i}\end{array}$ & $|\mathbf{F}(\mathbf{z})|$ \\
\hline 1 & 1.06 & 16 & 1.00 \\
\hline 2 & 1.63 & 17 & 1.00 \\
\hline 3 & 1.45 & 18 & 1.00 \\
\hline 4 & 1.29 & 19 & 1.00 \\
\hline 5 & 1.16 & 20 & 1.00 \\
\hline 6 & 1.04 & 21 & 1.00 \\
\hline 7 & 0.96 & 22 & 1.00 \\
\hline 8 & 0.92 & 23 & 1.00 \\
\hline 9 & 0.91 & 24 & 1.00 \\
\hline 10 & 0.94 & 25 & 1.00 \\
\hline 11 & 0.99 & 26 & 1.00 \\
\hline 12 & 1.02 & 27 & 1.00 \\
\hline
\end{tabular}




\begin{tabular}{|l|l|l|l|}
\hline 13 & 1.03 & 28 & 1.00 \\
\hline 14 & 1.03 & 29 & 1.00 \\
\hline 15 & 1.01 & 30 & 1.00 \\
\hline
\end{tabular}

Fig. 6: Orbit of $F(z)$ at $z_{0}=(-0.1+-0.4 i)$

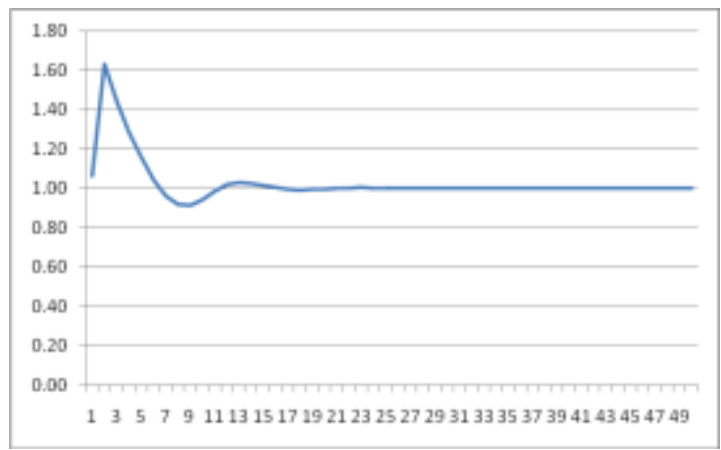

\section{Complex dynamics:}

\section{(i) Dynamics of Pell- Fibonacci function $F(z)$ :}

The dynamics of the orbit of $\mathrm{F}(z)$ at different points given in the Table 1 is shown in the Figures 4, 5 and 6. It is clear from the Fig 4 and the table attached that the orbit at $\mathrm{F}(z)$ at $\mathbf{z}_{0}=$ $\mathbf{0 . 8 2 2 6 7 9 0 2 9 9}+\mathbf{0 . 1 1 8 3 7 6 9 1 6 5 i}$ is an attracting Fixed point, whereas the orbit at $\left.\mathbf{z}_{0}=\mathbf{( - 0 . 0 4 9 0 1 7 7 0 2}, \mathbf{1 . 9 8 6 1 2 2 8 6 9} i\right)$ is the escaping points, see Fig. 5 and the table attached. Further, the orbit of $\mathbf{( - 0 . 1}, \mathbf{- 0 . 4 i )}$ leads to the fixed point i.e. $(1,0)$, see Fig. 6 and table attached. We apply the algorithm mentioned below to $F(z)$ of Pell-Fibonacci with $N=100$ and $M=4.0$. Fig. 7 shows the escape time where the red color region corresponds to rapid escape and the other hues, running from magenta corresponds to slow escape. There is a remarkable black region right at the centre in Fig 8 and many other smaller black regions in Fig 7. There are also the circular constructions of black regions.

\section{(ii) Dynamics of Pell- Lucas function $L(z)$ :}

The Pell-Lucas dynamics is studied for different points as given in Table 2. Here no fixed point is evaluated for the function $L(z)$. We applied the escape time algorithm mentioned below to function $L(z)$ of Pell-Lucas with $N=100$ and $M=4.0$. Fig. 9 here shows the escape time fractal where the red color region corresponds to rapid escape and the other hues, running from magenta to yellow corresponds to slow escape. But, we do not observe the existence of any black colored region in this fractal image and hence no fixed point.

\section{Escape time Algorithm:}

It refers to some region in complex plane and typical color is used to indicate the number of iterations before iterate gets large [13]. Escape time algorithm are employed to visualize the dynamics corresponding to the Julia sets and Mandelbrot sets.

The algorithm corresponding to $\mathrm{F}(z)$ or $L(z)$, to create the escape time criteria is as follows:

- Select a maximum iteration bound N, and a sense of unbounded $\mathrm{M}$.
For all pixels $(\mathrm{j}, \mathrm{k})$ corresponding to the points $\mathrm{z}$ in a rectangular portion of the complex plane, do the following:

- start

- $\quad$ let $i=0$

- While $z<M$ and $i<N$ do

$$
z=f(z)
$$$$
i=i+1
$$

end while

- If $i=N$, mark the pixel $(j, k)$ black, otherwise, mark the pixel a hue that corresponds to $i$.

- end

Fig. 7: Escape time Fractal of Pell- Fibonacci Sequence $F(z)$ for $-0.91 \leq \operatorname{Re}(z), \operatorname{Im}(z) \leq-1.07$

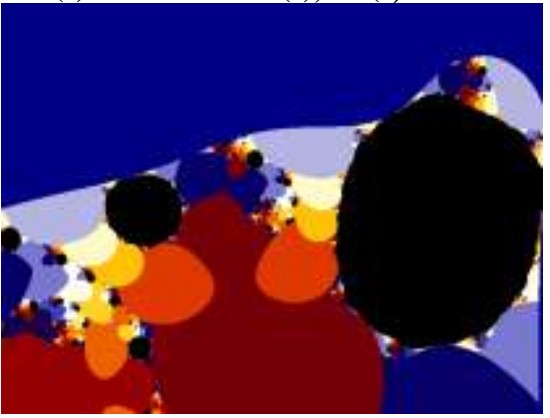

Fig. 8: Zoom of the Fig 7 that indicates the fixed point for Pell Sequence $F(z)$.

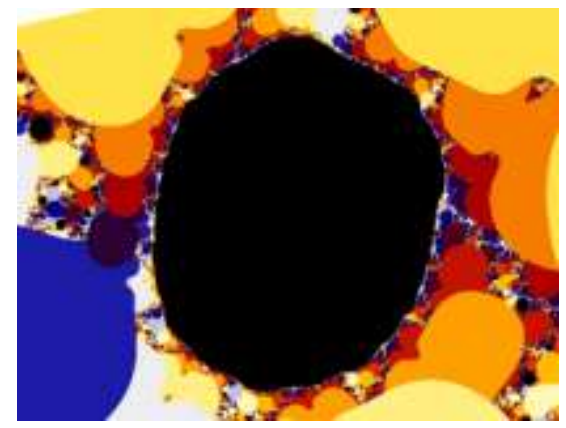

Fig. 9: Escape time Fractal of Pell- Lucas Sequence $L(z)$

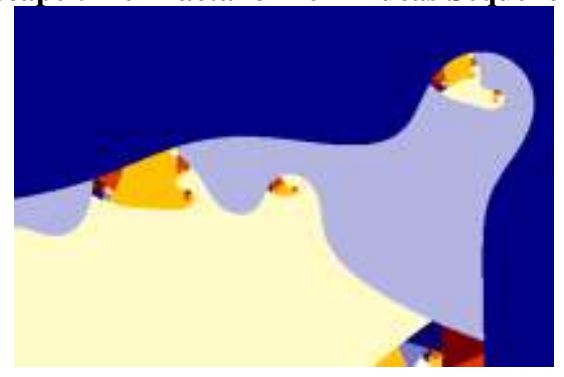

\section{Dynamics of Pell-Fibonacci and Pell-Lucas using Mann iterates:}

(i) Dynamics of Pell-Fibonacci function F(z): Using Manniterates, we find a change in the dynamics of Pell-Fibonacci function. Initially, we divide our Mandelbrot images into two 
groups, first when $\mathrm{s}=1$ (the special case) and second when $0<\mathrm{s}$ $<1$ (the general case).

Case I: $(s=1$, special case): The result of this special case is the usual Mandelbrot set which we obtained in Fig. 7, using above mentioned escape time algorithm with $\mathrm{N}=100$ and $\mathrm{M}=$ 4.0.

Case II: $(0<s<1$, general case): Here we get different Mandelbrot images for distinct values of $s$, We vary the values of $s$ from 0.1 to 0.8 and observe the variance in the Mandelbrot images and the fixed points.

Table 7: Table of Mann-Iterates of Pell-Fibonacci function $\mathbf{F}(z)$

\begin{tabular}{|c|c|c|c|c|c|c|c|c|c|c|}
\hline \multicolumn{2}{|r|}{$x$} & -4 & -3 & -2 & -1 & 0 & 1 & 2 & 3 & 4 \\
\hline $\begin{array}{l}\text { At } \\
s= \\
0.1\end{array}$ & $\mathbf{y}_{1}$ & -1.2 & 0.5 & 0.2 & 0.1 & 0 & 0.1 & 0.2 & 0.5 & 1.2 \\
\hline $\begin{array}{l}\text { At } \\
s= \\
0.3\end{array}$ & $\mathbf{y}_{2}$ & -3.6 & 1.5 & -0.6 & 0.3 & 0 & 0.3 & 0.6 & 1.5 & 3.6 \\
\hline $\begin{array}{l}\text { At } \\
s= \\
0.5\end{array}$ & $\mathbf{y}_{\mathbf{3}}$ & -6 & 2.5 & -1 & 0.5 & 0 & 0.5 & 1 & 2.5 & 6 \\
\hline $\begin{array}{l}\text { At } \\
s= \\
0.8\end{array}$ & $\mathbf{y}_{4}$ & -9.6 & 4 & -1.6 & 0.8 & 0 & 0.8 & 1.6 & 4 & 9.6 \\
\hline $\begin{array}{l}\text { At } \\
s= \\
1\end{array}$ & $\mathbf{y}_{5}$ & -12 & 5 & -2 & 1 & 0 & 1 & 2 & 5 & 12 \\
\hline
\end{tabular}

The above table of Pell-Fibonacci function for Mann-Iterates shows that as the value of $s$ varies from 0.1 to 1 , the number of fixed points increases and their distinctness is observed. We observe more fixed points as we approach closer to 1 , while as we move towards 0 , we have greater escape region corresponding to fixed point 0 . The black colored region grows larger and larger as one approach nearby 0 . This is clearly observed after viewing figures 10,11, 12 and 13 respectively. Thus, Mann-iterates gives us a wide range of escape time fractals of Pell-Fibonacci varying for different values of $s$.

Escape time Algorithm: The escape time algorithm is employed to visualize the dynamics corresponding to the Julia sets and Mandelbrot sets [13]. Here we used the above mentioned algorithm for Pell-Fibonacci function with $N=100$ and $M=4.0$, for different values of $s$.

Fig. 10: Escape time fractal for Mann iterate $s=0.1$

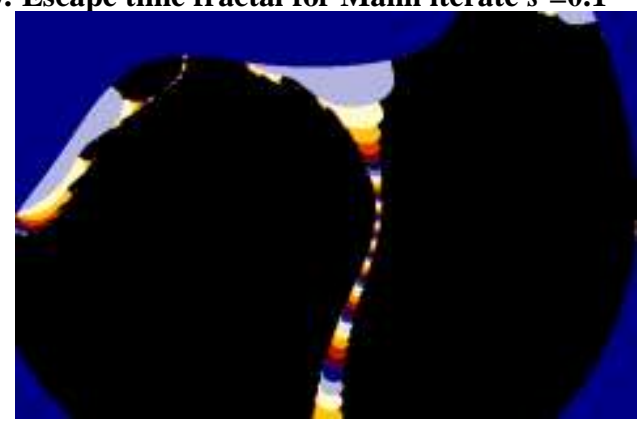

Fig. 11: Escape time fractal for Mann iterate $s=0.3$

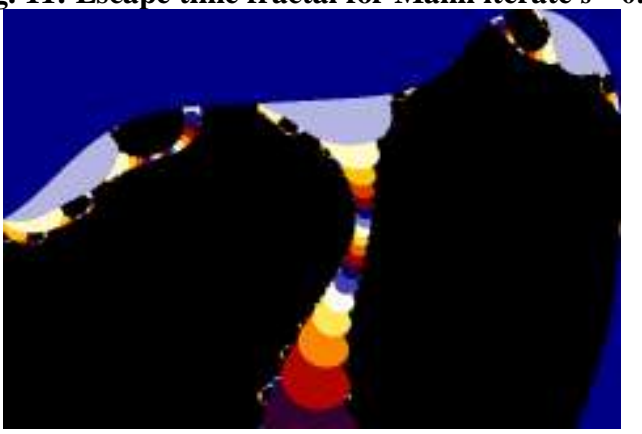

Fig. 12: Escape time fractal for Mann iterate $s=0.5$

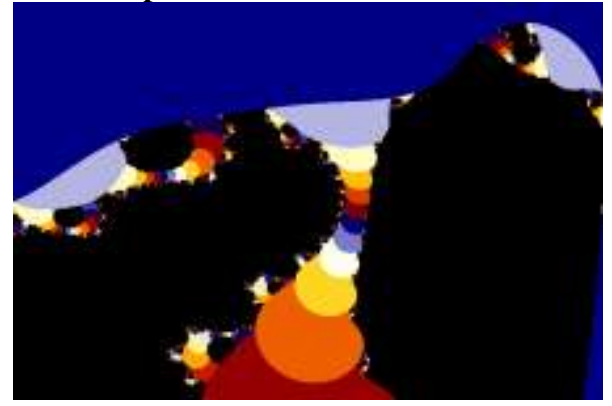

Fig. 13: Escape time fractal for Mann iterate $s=0.8$

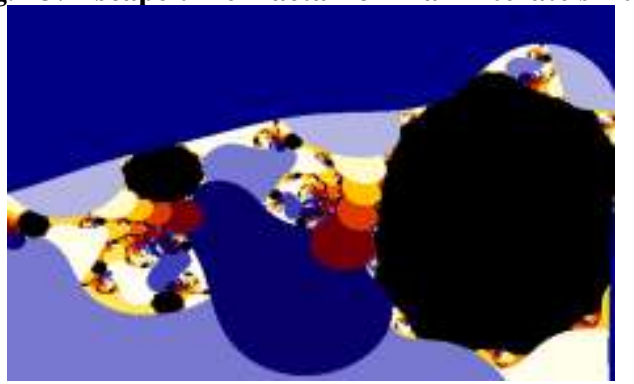

(ii) Dynamics of Pell-Lucas function $\mathbf{L}(z)$ : Using Manniterates, we find a major change in the dynamics of Pell-Lucas function. Here also, we divide our Mandelbrot images into two groups.

Case I: ( $s=1$, special case): The result of this special case is the usual Mandelbrot set which we obtained in Fig. 9, using above mentioned escape time algorithm with $\mathrm{N}=100$ and $\mathrm{M}=$ 4.0.

Case II: $(0<s<1$, general case): Here we get different Mandelbrot images for distinct values of $s$, We vary the values of $s$ from 0.1 to 0.8 and observe the variance in the Mandelbrot images and the fixed points.

Escape time Algorithm: Here we used the algorithm mentioned previously for special case of Pell- Lucas function with $N=100$ and $M=4.0$, for different values of $s$. As a result we get different fractal images corresponding to Pell- Lucas function. 
Table 8: Table of Mann-Iterates of Pell- Lucas function L $(z)$.

\begin{tabular}{|c|c|c|c|c|c|c|c|c|}
\hline \multicolumn{2}{|c|}{$\boldsymbol{x}$} & $\mathbf{- 3}$ & $\mathbf{- 2}$ & $\mathbf{- 1}$ & $\mathbf{0}$ & $\mathbf{1}$ & $\mathbf{2}$ & $\mathbf{3}$ \\
\hline $\begin{array}{c}\text { At } \boldsymbol{s} \\
\mathbf{0 . 0 1}\end{array}$ & $\mathbf{y}_{\mathbf{1}}$ & -0.14 & 0.06 & -0.02 & 0 & 0.02 & 0.06 & 0.14 \\
\hline $\begin{array}{c}\text { At } \boldsymbol{s}= \\
\mathbf{0 . 1}\end{array}$ & $\mathbf{y}_{\mathbf{2}}$ & -1.40 & 0.6 & -0.20 & 0.20 & 0.20 & 0.6 & 1.40 \\
\hline $\begin{array}{c}\text { At } \boldsymbol{s}= \\
\mathbf{0 . 3}\end{array}$ & $\mathbf{y}_{\mathbf{3}}$ & -4.20 & 1.80 & -0.60 & 0.60 & 0.60 & 1.80 & 4.20 \\
\hline $\begin{array}{c}\text { At } \boldsymbol{s}= \\
\mathbf{0 . 4}\end{array}$ & $\mathbf{y}_{\mathbf{4}}$ & -5.6 & 2.4 & -0.8 & 0.8 & 0.8 & 2.4 & 5.6 \\
\hline $\begin{array}{c}\text { At } \boldsymbol{s}= \\
\mathbf{0 . 5}\end{array}$ & $\mathbf{y}_{\mathbf{5}}$ & -7.00 & 3.00 & -1.00 & 1.00 & 1.00 & 3.00 & 7.00 \\
\hline $\begin{array}{c}\text { At } \boldsymbol{s}= \\
\mathbf{0 . 6}\end{array}$ & $\mathbf{y}_{\mathbf{6}}$ & -8.4 & 3.6 & -1.2 & 1.2 & 1.2 & 3.6 & 8.4 \\
\hline $\begin{array}{c}\text { At } \boldsymbol{s}= \\
\mathbf{0 . 8}\end{array}$ & $\mathbf{y}_{7}$ & -11.2 & 4.8 & -1.6 & 1.6 & 1.6 & 4.8 & 11.2 \\
\hline $\begin{array}{c}\text { At } \boldsymbol{s}= \\
\mathbf{1 . 0}\end{array}$ & $\mathbf{y}_{\mathbf{8}}$ & -14 & 6.00 & -2 & 2 & 2 & 6 & 14 \\
\hline
\end{tabular}

From the Table 8 , it is observed that we have the existence of fixed points ranging from $s=0.01$ to $s=0.6$. When we approach towards mid-values i.e. at $s=0.6, s=0.5, s=0.4$ and $\quad s=0.3$, we find more number of fixed points. The black colored region grows larger and larger as one approach nearby 0 . This is clearly observed after viewing figures 14, 15, 16, 17, 18 and 19. Earlier we do not get any fixed point for Pell-Lucas function but Manniterates gave us a wide range of beautiful Pell-Lucas dynamics ranging from $s=0.1$ to $s=0.8$.

Fig. 14: Escape time fractal for Mann iterate $s=0.01$

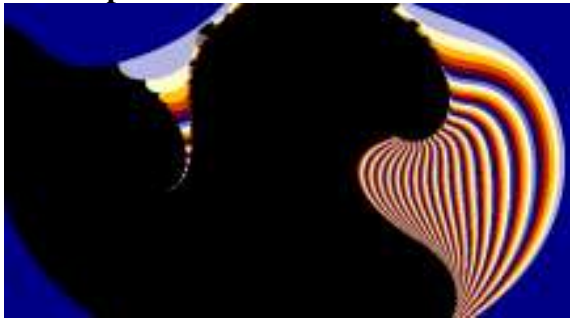

Fig. 15: Escape time fractal for Mann iterate $s=0.1$

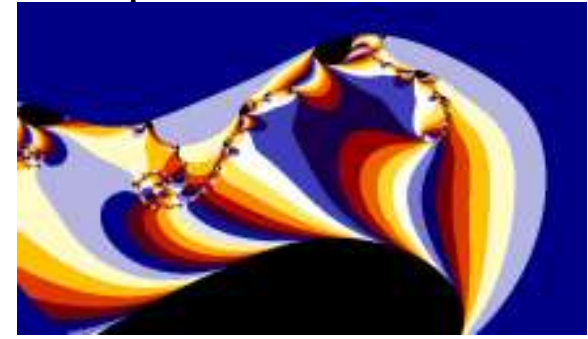

Fig. 16: Escape time fractal for Mann iterate $s=0.3$

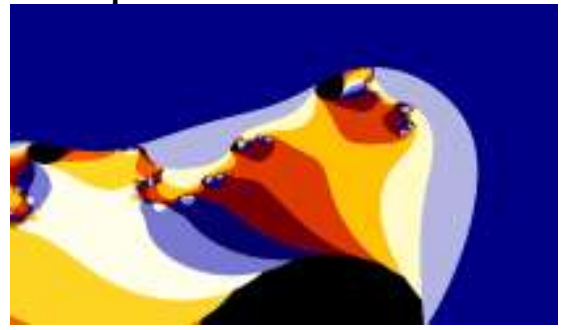

Fig. 17: Escape time fractal for Mann iterate $s=0.4$

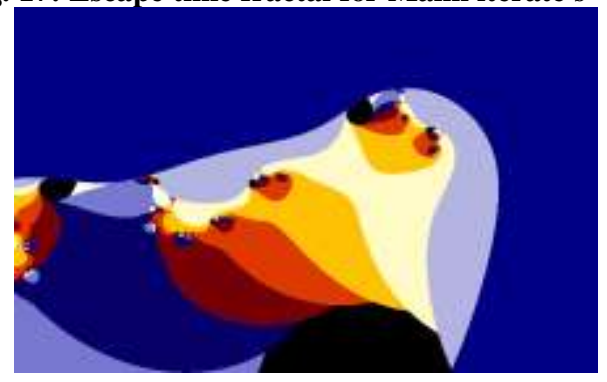

Fig. 18: Escape time fractal for Mann iterate $s=0.5$

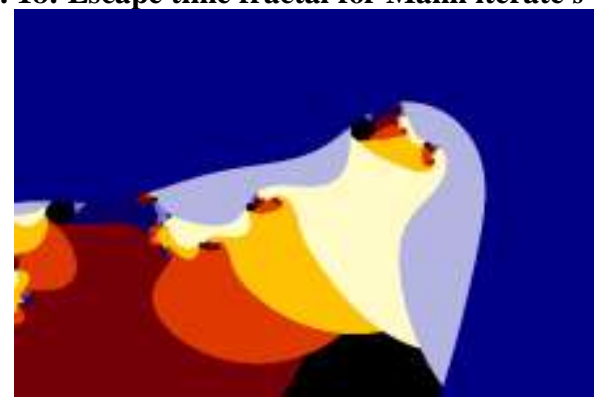

Fig. 19: Escape time fractal for Mann iterate $s=0.6$

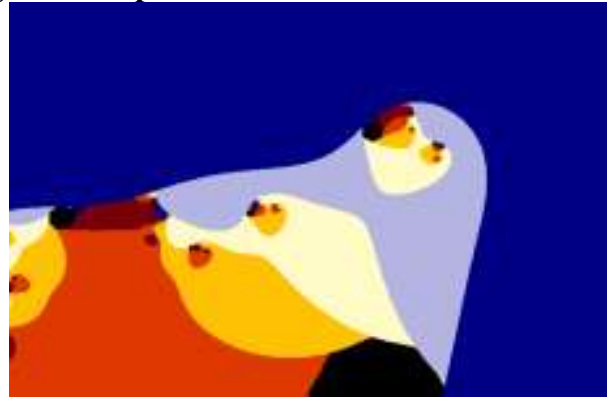

\section{Conclusion:}

In our study, the complex dynamics of Pell-Fibonacci and PellLucas numbers have been investigated using Binet type formula. In Pell-Fibonacci fractal, we find that there are integer fixed points which are associated with a large basin of attraction. Besides these, there are also some additional complex fixed points and the escape time images show that the Pell-Fibonacci numbers possesses a rich complex dynamics. Whereas, a comparative study on Pell-Lucas fractal image shows that there is no existence of any fixed point for Pell- Lucas function. We also applied Mann-iterates to investigate a new dynamics of both functions. Pell-Fibonacci showed its rich dynamics for Manniterates rendering different Mandelbrot images at different values. On the other hand Pell-Lucas function shows a major change in its dynamics by showing the existence of its fixed points at different Mann-iterates values, thereby verifying its richness of complex dynamics.

\section{REFERENCES}

[1] Barnsley M. F., "Fractals Everywhere", New York Academic Press, (1998). 
[2] Bicknell, M., "A primer of the Pell sequence and related sequences", The Fibonacci Quarterly, 13(4) (1975), 345349.

[3] Binet M. J., "Memoire surl' integration desequations linearies aux differences finies, d'un order quelconque, a coefficients variables", Comptes Rendus DES Seances de L'academic des sciences 17(1843), 559-565.

[4] Carslon, P. W, "3D rendering methods for fractals in the complex plane", Computer and Graphics, 20(5),(1996), 757-758.

[5] Clifford, A. R., "Views of Fibonacci dynamics", Computer and Graphics 28(2004), 297-300.

[6] Devaney R., "Chaos, Fractals and dynamics, Computer experiments in mathematics", Menlo Park:AddisonWessley, (1990).

[7] Dickson L. E., "History of the theory of numbers", Vol I. New York: Chessa Publishing Company, reprint 1971.

[8] John, C, "A new class of q-Fibonacci polynomials", The Electronic journal of combinatorics 10 (2003), \#R1 9, MR Subject Classification : primary 05A30, 05A15; secondary: $15 \mathrm{~A} 15$.

[9] W.R.Mann, "Mean Value methods in iteration", Proc. Amer. Math. Soc.4 (1953), 506-510.

[10] Kalman D. and Mena R., "The Fibonacci numbers Exposed", Math Magazine, 76:3(2003), 167-181.

[11] Kohsy T.,"Fibonacci and Lucas numbers with Applications", John Wiley and Sons, NY, (2001).
[12] Melham, R., "Sums involving Fibonacci and Pell numbers", Portugaliae Mathematica, Vol. 56 Fasc. 3 (1999)

[13] Mustapha R.; Saeki, O, "Extending generalized Fibonacci sequences and their Binet type formula", 2000 Mathematics Subject Classification. Primary 40A05; Secondary 40A25.

[14] Peitgen H. O. and Ritcher PH, "The beauty of Fractals", Berlin :Springer, (1986)

[15] Reiter CA, "Fractals, visualizations and J", $2^{\text {nd }}$ ed. Toronto: Jsoftware Inc.(2000).

[16] Senechal M., "Quasi crystals and geometry", New York: Cambridge Univ. Press, (1995).

[17] Stojmenovic, I, "Recursive Algorithms in Computer Science Courses: Fibonacci Numbers and Binomial Coefficients", IEEE Transactions on Education, Vol. 43, No. 3, August (2000)

[18] Vsemirnov, M, "A New Fibonacci-like Sequence of Composite Numbers", Article 04.3.7 Journal of Integer Sequences, Vol. 7 (2004).

\section{Web References}

[1] Jsoftware, http://www.jsoftware.com.

[2]

Fibonacci

Association: http://www.mcs.dal.ca/Fibonacci/fibnat.html

[3] Reiter CA, Escape time zones of the Fibonacci numbers, http://www.lafayette.edu/ reiterc/mvp/fib_v/index.html. 